Published in final edited form as:

Anal Chem. 2019 May 07; 91(9): 6180-6189. doi:10.1021/acs.analchem.9b00822.

\title{
Highly Sensitive O-Glycan Profiling for Human Serum Proteins Reveals Gender-Dependent Changes in Colorectal Cancer Patients
}

\author{
Solomon T. Gizaw ${ }^{1,+,{ }^{*}, \text { Stefan Gaunitz }}{ }^{1,+, \#}$, and Milos V. Novotny ${ }^{1,{ }^{* *}}$ \\ ${ }^{1}$ Department of Chemistry, Indiana University, Bloomington, Indiana 47405
}

\begin{abstract}
A newly developed microscale protocol for profiling serum O-glycans has been validated here with multiple serum samples obtained from different cohorts of colorectal cancer patients. The simultaneous cleavage and permethylation steps in this procedure preserve the integrity of released minor O-glycans, so that 39 O-linked oligosaccharides could be reliably recorded in a profile. This is far more detected components than shown in any previous studies. The analytical results were further subjected to a battery of statistical tests. Our O-glycan compositions compare favorably with the previous results obtained with solid tumors and cancer cell lines, suggesting that smaller circulatory mucins protruding into the blood circulation may be one source of O-glycans that we observe in the serum samples. While the control vs. cancer statistical comparisons generally agree with the expected glycosylation trends, the comparisons of male vs. female subjects have led to some surprising results for which we do not have a ready explanation due to lack of any literature describing hormonal control of O-glycosylation. Our results thus underscore the necessity of applying new analytical technologies to clinically interesting sample sets.
\end{abstract}

\section{Graphical Abstract}

\footnotetext{
${ }^{* *}$ Corresponding Author novotny@indiana.edu.

${ }^{+}$Contributed equally to this work

* Current address: Department of Biochemistry, School of Medicine, College of Health Sciences, Addis Ababa University, Addis Ababa 9086, Ethiopia

\#Current address: KTH Royal Institute of Technology, School of Engineering Sciences in Chemistry, Biotechnology and Health, Division of Glycoscience/Department of Chemistry, AlbaNova University Center SE-106 91 Stockholm, Sweden

The authors declare no competing financial interest.
} 


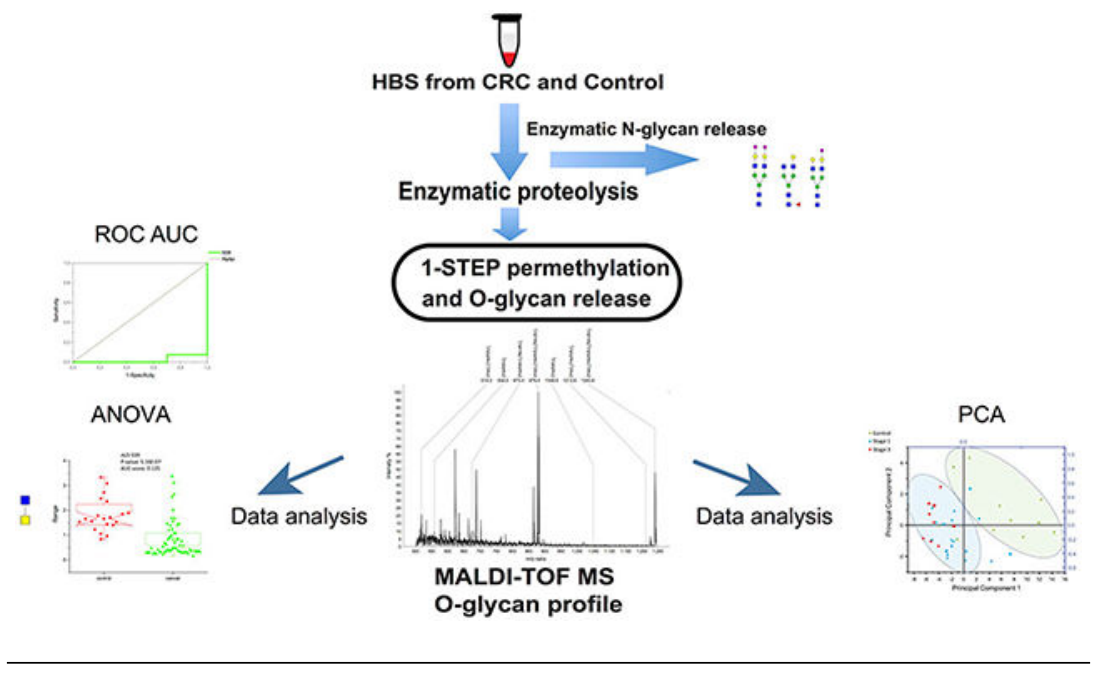

Many clinically relevant analyses now utilize human blood serum or plasma for both the routine clinical measurements and various efforts to look for potential disease biomarkers. Glycoproteins are among the most targeted biomarker molecules. Aberrant glycosylation of proteins in different types of cancer has provided stimulus for numerous studies for already several decades ${ }^{1-3}$, but detection of definitive glycoconjugate cancer biomarkers in blood still remains somewhat elusive. At present, there are many efforts to utilize relatively new technologies, such as glycan and lectin arrays, and mass spectrometry (MS)-based analytical techniques for glycomic profiling ${ }^{4-7}$. MS and its ancillary techniques have now been recognized to have the unparalleled capabilities and potential in providing definitive structural information on glycoconjugate attributes of malignant cellular transformation.

The proteins in blood span a very large range of concentrations ${ }^{8}$, while more than $50 \%$ of these proteins appear glycosylated at asparagine or serine/threonine residues and rarely at other sites. At this time, the glycan profiling techniques have been far more successfully developed for asparagine ( $\mathrm{N}$-linked) oligosaccharides than the serine/threonine (O-linked) glycans, as exemplified by the recent applications to cancer research ${ }^{9-13}$. This situation is primarily due to the availability of a reliable enzymatic cleavage for the former glycan type, which cannot be readily achieved for O-glycans. With O-glycans, many investigators still rely on the classical beta-elimination procedure ${ }^{14}$, facilitated through alkaline chemical cleavage, or its more recent modifications toward the glycan analysis. The main drawbacks of this approach are: (a) the need to use relatively large amounts of biological material; and (b) perhaps most importantly, a danger of degrading O-glycan structures during the so-called "peeling reactions". Consequently, most attempts at profiling report only a limited number of major O-glycans rather than their more complete profiles ${ }^{15}$. This situation has called for substantial procedural improvements for some time, as underscored by the obvious functional importance of large mucinous proteins in relation to cancer $1,16-18$

As demonstrated in this communication, we can now successfully detect a wide range of Oglycan structures through a modified and miniaturized version of our previously reported procedure $^{19}$, where the enzymatically degraded proteins were subjected to a cleavage during the following permethylation step. As shown below, only small volumes of blood serum 
(low microliters) are needed in these determinations. In order to enhance profiling selectivity for O-glycans, we first remove potential interferences from $\mathrm{N}$-linked oligosaccharides by their prior enzymatic cleavage with $\mathrm{N}$-glycanase. The simultaneous O-glycan cleavage and permethylation is an inherently advantageous feature of this procedure, in which we protect the minor glycans that could otherwise be lost due to the peeling reactions and formation of other procedural artifacts. This has allowed us to record fairly comprehensive profiles of serum O-glycans during this study.

For the initial validation of our O-glycan profiling procedure using MALDI-MS, we have chosen the set of samples from colorectal cancer patients. These very same samples were initially collected for the benefits of $\mathrm{N}$-glycan profiling, as previously described in this journal ${ }^{20}$.

Colorectal cancer (CRC) is one of the most common oncological diseases with sporadic causes, high tendency toward metastasis and relatively high mortality ${ }^{21,22}$. The glycosylation events in colon cancer were recently reviewed extensively by Holst et al. ${ }^{23}$. Several hallmark aberrations due to this disease are quite similar to what has been observed in several other cancers, including but not limited to, an increased $\beta 1-6$ branching and poly$\mathrm{N}$-acetyl-lactosamine extension in $\mathrm{N}$-glycan structures, increase in truncated paucimannosidic structures and a decrease of bisecting $\mathrm{N}$-acetyl-lactosamine (LacNAc) structures. The changes in O-glycosylation can include decrease in Core 3 and Core 4 structures and increased levels of structures such as (sialyl) Tn and (sialyl) T antigen. Increases in sialylation, fucosylation and occurrence of the Lewis-type antigens and Type 2 chains have been indicated in tissue biopsies and cancer cell lines. Considerable evidence points to modifications of short-chain O-glycans (known as " $\mathrm{T}$ ", "Tn" and sialylated $\mathrm{Tn}$ antigens) on the serial sections of mucin-based serine and threonine residues ${ }^{24}$, but only a limited number of actual glycans have been structurally indicated. In contrast, in our report, around 40 oligosaccharides can now be routinely assessed quantitatively, providing the basis for statistically evaluating different cohort groups of colorectal cancer patients.

The O-glycan profiles from 62 patients' serum samples (both males and females at different cancer stages, (referred here Cycle 1 and 2) and 20 healthy control individuals provided the basis for statistical evaluations: one-way ANOVA analysis; principal component analysis (PCA); and Receiver Operating Characteristics (ROC) evaluation plots for selected Oglycans. Five O-glycans were significantly altered in cancer (Cycle 1 and 2 combined) at the 0.05 level. The changes in glycan profiles correlated with cancer progression, but also importantly and unexpectedly, we observed gender differences in these O-glycan profiles. To our knowledge, this has not been reported previously. Glycosylation changes due to cancer could be expected in different forms (either lower or higher expression of certain structures, incomplete glycosylation, or even a possibility of previously unobserved structures), however, it is commonly believed that these changes do not occur at random ${ }^{1}$. With the possibility of reproducibly profiling a large number of glycans, seemingly complex process of disordered glycosylation in cancer can now be increasingly approached. This study further underscores the great untapped information on O-glycosylation and its role in cancer as indicated by the close correlation between altered glycan profiles and cancer progression. 


\section{EXPERIMENTAL SECTION}

\section{Chemicals and Reagents}

Beta-mercaptoethanol, sodium hydroxide beads, methyl iodide, trypsin (EC 3.4.21.4, TPCKtreated from bovine pancreas) were purchased from Sigma-Aldrich (St. Louis, MO). 2,5Dihydroxybenzoic acid (2,5-DHB) was received from Alfa Aesar (Ward Hill, MA). NGlycanase (PNGase F) was purchased from Prozyme (Hayward, CA), while Pronase (from Streptomyces griseus) was a product of Roche Diagnostics (Indianapolis, IN), as was Nonidet P-40. LC-MS grade water and acetonitrile were purchased from EMD Chemicals (Gibbstown, NJ). Trifluoroacetic acid (TFA), glacial acetic acid, formic acid (FA), chloroform, and N,N'-dimethylformamide (DMF) were products of Mallinckrodt Baker (Phillipsburg, NJ). Micro SpinColumn Empty and Ultra-Micro SpinColumn amino columns were purchased from Harvard Apparatus (Hayward, CA), while Amicon Ultra-0.5 Centrifugal Filter Unit with Ultracel-10 membrane was from EMD Millipore (a part of Millipore Sigma). Sodium dodecylsulfate (SDS) was acquired from Biorad (Hercules, CA).

\section{Clinical Specimens}

The human blood serum samples investigated in this study were obtained from Hoosier Oncology Group, Inc. (Indianapolis, IN) as a part of sample collection targeted to provide specimens to investigators pursuing different biomarker research objectives. The Walther Cancer Institute associated with the Indiana University School of Medicine facilitated coordination of the sample collection effort. The study was approved by the institutional ethics committee of Indiana University and performed in accordance with the ethical standards of the Helsinki Declaration. Blood samples were collected from the various patients diagnosed with various stages and types of colorectal cancer $(n=62)$ as well as healthy control individuals $(\mathrm{n}=20)$. Blood was collected into sterile vacutainer tubes and allowed to clot for $30 \mathrm{~min}$ at ambient temperature, then subjected to centrifuging, aliquoting and freeze and storage at $-80^{\circ} \mathrm{C}$. All samples were deidentified to protect the sample donors' privacy.

All colorectal cancer patients were subjected to blood collection after their primary cancer treatment was finished (further referred to as "Cycle 1" in our text) and at the time of patients' relapse (Cycle 2). There were 20 male patients and 18 female patients in Cycle 1 and 15 males and 9 females in Cycle 2. The colorectal cancer patients under this study underwent a variety of individualized treatments, including different types of chemotherapy with single or multiple agents, radiation therapy and surgery. For statistical comparison, all samples were divided according to their gender and "cycles". Although there were some differences in race, ethnicity and smoking status, the number of cases were insufficient for statistical evaluations.

\section{Serum O-Glycan Measurements}

O-Glycans were extracted from the human blood serum (HBS) of colorectal cancer patients and healthy individuals as based on our previously reported method ${ }^{19}$ with minor modifications regarding the proteolysis since the first publication. Briefly, HBS $(2.5 \mu \mathrm{L})$ was denatured by $25 \mu \mathrm{L}$ of $0.1 \%$ SDS and $0.1 \% \beta$-mercaptoethanol in $10 \mathrm{mM}$ sodium phosphate 
(pH 7.5) at $60^{\circ} \mathrm{C}$ for $1 \mathrm{~h}$. After the samples were cooled for $10 \mathrm{~min}$ at room temperature, 2.5 $\mu \mathrm{L}$ of $10 \%$ Nonidet P-40 (NP-40) was added and allowed to equilibrate for $10 \mathrm{~min}$. Subsequently, $1 \mu \mathrm{L}$ of $0.25 \mathrm{mU}$ of PNGase $\mathrm{F}$ in $10 \mathrm{mM}$ sodium phosphate (pH 7.5) was added to each sample and incubated at $37^{\circ} \mathrm{C}$ for $21 \mathrm{~h}$. N-glycans were removed by size exclusion through Amicon ${ }^{\circledR}$ Ultra-0.5 (10 KDa) (flow through). To separate the proteins from N-glycans, 10,000 MWCO filters (Amicon Ultra Centrifugal filters $0.5 \mathrm{~mL}$, Millipore, Billerica, MA) were prepared by adding $400-\mu \mathrm{L}$ water and centrifuging for $10 \mathrm{~min}$ at 9300 $\times \mathrm{g}$, followed by $400-\mu \mathrm{L} 10-\mathrm{mM}$ sodium phosphate buffer $(\mathrm{pH} 7.5$ ) and centrifuging for 10 $\mathrm{min}$ at $9,300 \times \mathrm{g}$. Subsequently, 300- $\mathrm{L} \mathrm{L} 10-\mathrm{mM}$ sodium phosphate buffer $(\mathrm{pH} 7.5)$ was first added to the centrifugal filters and then the PGNase F-digested sample $(50 \mu \mathrm{L})$ was added and mixed by pipetting. The centrifugal filters were centrifuged for $15 \mathrm{~min}$ at 14,000 $\times \mathrm{g}$, followed by addition of $2 \times 400-\mu \mathrm{L} 10-\mathrm{mM}$ sodium phosphate buffer ( $\mathrm{pH} 7.5$ ), and centrifuged at 14,000 $\times \mathrm{g}$ for $15 \mathrm{~min}$. The first flow-through and sodium phosphate buffer flow-through containing the N-glycans were pooled and dried with a CentriVap concentrator. The centrifugal filters were then turned upside down and centrifuged for $15 \mathrm{~min}$ at 14,000 $\times g$ to elute the proteins. The protein fractions were continued in the O-glycan preparation as follows.

The O-glycosylated peptides were purified by amino Ultra SpinColumns (Harvard Apparatus). The columns were washed $3 \times$ with buffer B (10\%/90\%/0.1\% acetonitrile, water, trifluoroacetic acid) and $3 \times$ with buffer A (90\%/10\%/0.1\% acetonitrile, water, trifluoroacetic acid) and centrifuged for $1 \mathrm{~min}$ at $200 \times \mathrm{g}$. Two hundred fifty-microliter volumes of buffer $\mathrm{A}$ were added to the digested protein samples and vortexed before applying samples to the columns. The columns were centrifuged for $1 \mathrm{~min}$ at $200 \times \mathrm{g}$; the flow-throughs were collected and reapplied to the column. The samples were reapplied and centrifuged two more times to ensure retention on the column. Following that, the columns were washed with $2 \times 300-\mu \mathrm{L}$ buffer A and centrifuged for $1 \mathrm{~min}$ at $200 \times \mathrm{g}$. The O-glycopeptide fractions were eluted by adding $300-\mu \mathrm{L}$ buffer $\mathrm{B}$, centrifuged at $200 \times \mathrm{g}$ for $\sim 10 \mathrm{~s}$, left equilibrating for $10 \mathrm{~min}$, then centrifuged for $1 \mathrm{~min}$ at $200 \times \mathrm{g}$. Another $300-\mu \mathrm{L}$ buffer B was added and centrifuged for $1.5 \mathrm{~min}$ at $800 \times \mathrm{g}$. The two buffer B eluate fractions were pooled and dried by a CentriVap concentrator. The O-glycopeptides were permethylated, in which the permethylation was also used here to release the O-glycans from the peptides and the glycans were thus not reduced first.

\section{Permethylation}

Permethylation was carried out as follows. First, the permethylation reactors were created by taking a 20-mL glass vial, filled with roughly one fourth with $\mathrm{NaOH}$ beads, and followed with acetonitrile. By using a cut $1-\mathrm{mL}$ pipette tip, the $\mathrm{NaOH}$ beads were transferred to empty Micro SpinColumns (Harvard Apparatus). Approximately three fourths of the column volume was filled up with the $\mathrm{NaOH}$ beads. The columns were centrifuged at $200 \times \mathrm{g}$ for 1 min to remove acetonitrile and then washed with $3 \times \sim 300-\mu \mathrm{L}$ DMF (dimethylformamide) and centrifuged for $1 \mathrm{~min}$ at $200 \times \mathrm{g}$. The not-reduced O-glycans from the peptides were supplemented with $5-\mu \mathrm{L}$ water, vortexed, and centrifuged for $30 \mathrm{~s}$ at $600 \times \mathrm{g}$. Next, $65-\mu \mathrm{L}$ DMF and $35-\mu \mathrm{L}$ methyl iodide were added to the samples and mixed. The samples were applied onto the permethylation reactors, and the reactors were put in the original sample 
centrifugation tubes, which were sealed by putting a cap on them. The reactors were set horizontally, slightly elevated, and left to react for $20 \mathrm{~min}$. Afterwards, the caps were removed and the permethylation reactors were centrifuged for $1.5 \mathrm{~min}$ at $200 \times \mathrm{g}$. The flowthroughs were reapplied to the reactors after $35-\mu \mathrm{L}$ methyl iodide was added and mixed. Caps were used to seal the reactors and they were set horizontally, slightly elevated, and left to react for another $20 \mathrm{~min}$. The caps were removed and reactors were centrifuged for 1.5 $\min$ at $2,300 \times \mathrm{g}$. Then, $400-\mu \mathrm{L}$ chloroform and $1-\mathrm{mL} 0.5-\mathrm{M} \mathrm{NaCl}$ were added to the flowthroughs and shaken gently by hand for $2 \mathrm{~min}$ before centrifuging the samples at $600 \times \mathrm{g}$ for $30 \mathrm{~s}$. The upper aqueous phases were discarded and 1-mL water was added to the lower chloroform phases. The mixtures were gently shaken by hand for $2 \mathrm{~min}$ and centrifuged for $30 \mathrm{~s}$ at $600 \times \mathrm{g}$. The upper aqueous phases were discarded again, while $1-\mathrm{mL}$ water was added and gently shaken by hand for $2 \mathrm{~min}$. The mixtures were centrifuged for $30 \mathrm{~s}$ at 600 $\times g$ and the upper aqueous phases were discarded. The chloroform phases were dried in a CentriVap concentrator and stored in a freezer until analysis.

\section{Data Collection and Analysis}

All the O-glycan profiles were recorded using MALDI-TOF/TOF mass spectrometry using an Applied Biosystems 4800 TOF-TOF instrument. The laser intensity was set at 4100, light intensity of 20, DIE 500, and mass range (500-3000). Briefly, the permethylated samples (dissolved in 1:1 methanol: water) were spotted and dried in room temperature (RT) on the plates for $10 \mathrm{~min}$ and DHB matrix (10 $\mathrm{mg} / \mathrm{mL}$ in 1:1 methanol: water), while a final concentration of 1-mM sodium acetate was added to each spot and dried again under vacuum for $2 \mathrm{~min}$. For each sample, three spectra were recorded. The spectra were baselinecorrected and noise-filtered (0.7) in the Applied Biosystems Data Explorer software (4.9) before export to text file format. The text files were uploaded to the ExPASy online tool GlycoMod that can predict the possible oligosaccharide structures from their experimentally determined masses. In GlycoMod, the UniCarbKB database was used for peak identification (+/- 0.2 Dalton filter was used for peak identification) and the structure/composition with a traceable UniCarbKB link were selected for constructing the O-glycan library of the study. A list of 39 oligosaccharide monoisotopic masses was made for the in-house developed program PeakCalc. The relative abundance and associated standard deviation of each peak was calculated with PeakCalc (+/- 0.5 Dalton filter was used for peak identification). Relative abundance (normalization) was calculated by dividing the intensity of each peak with the total intensity of all 39 O-glycans. Triplicate sample files exported from PeakCalc in CSV data format were merged and imported into Microsoft Excel for rendering of plots and sorting of data.

\section{Statistical Tests}

Advanced statistical tests such as one-way ANOVA, Principal Component Analysis (PCA) and Receiver Operating Characteristics (ROC) Area Under the Curve (AUC) plots were performed with OriginPro2018 (9.5.1.195) on the normalized MS intensity data imported from Microsoft Excel sheets. Briefly, the one-way ANOVA was performed pairwise with control vs Cycle 1 or Cycle 2 with 0.05 significance level. The means comparisons were performed with Tukey test at the 0.05 significance level, while box plots were chosen for the 
output format. The p-values of the one-way ANOVA reported in this communication refer to the means comparison with the Tukey test, not the F-value of the ANOVA.

\section{RESULTS AND DISCUSSION}

Aberrant glycosylation of cellular surfaces in tumorigenesis and metastatic promotion has been a subject of numerous studies for quite some time ${ }^{1-3}$. New analytical techniques that improve our structural knowledge of glycan antigens are becoming increasingly important for diagnostic, prognostic and even therapeutic purposes ${ }^{25,26}$. Most contemporary knowledge of O-glycosylation in cancer has been primarily obtained from cancer cell lines and tumor tissue analyses ${ }^{23,27}$, and much less frequently from the analyses of body fluids such as blood serum or plasma ${ }^{23}$ Moreover, structural considerations of O-glycans have often been deduced from transcriptomic data ${ }^{31}$ rather than direct measurements. Whereas $\mathrm{N}$ glycan profiling in serum samples, for the sake of biomarker discovery, has seen some progress during the recent years ${ }^{9-13}$, sufficiently sensitive analytical techniques for serum Oglycans have been rare and limited to a few isolated structures, ${ }^{15,28,30,32-34}$ some of which may even be degradation products of uncontrolled deglycosylation. This is, to a large extent, due to unavailability of the "cleaner" glycan release protocols, which caused various investigators to resort to a chemical cleavage from serine and threonine residues in the protein backbone with its earlier mentioned methodological disadvantages.

The modification and miniaturization of the previously reported procedure ${ }^{19}$, as shown in this work, leads us to a substantially enhanced profiling capability, now including numerous O-glycans from low-microliter volumes of serum samples. After enzymatic removal of the $\mathrm{N}$-glycans and complete proteolysis, the $\mathrm{O}$-glycans were released and permethylated in one step. This allowed us to record about $40 \mathrm{O}$-glycan components within a substantial concentration range, as seen in the Excel plot of MALDI-MS data shown in Figure 1. With the expected repeatability of the MALDI-MS-based measurements (approximately $\pm 15 \%$ variation), the large standard deviation error bars very likely reflect substantial variations due to a disease state and possibly gender. A dot plot of the same data set is shown in Figure S1 (supporting information). A typical raw spectrum of the serum-originated components (Figure S2) and the O-glycan annotated spectral profile (Figure S3) are also shown in the Supporting Information. Table 1 further specifies the individual $\mathrm{m} / \mathrm{z}$-ratios together with the glycan compositions, which are very likely due to a close correspondence between the measured and expected masses. Besides the few main O-glycans that are usually seen in biological specimens (that is $\mathrm{m} / \mathrm{z} 879,1241)$, minority peaks $(<10 \%)$ have now been reproducibly recorded here as well, primarily due to the fact that their structures are preserved with minimal degradation, as the cleavage and permethylation occur rapidly at the same time. To validate our protocol, we have chosen the sets of samples from colorectal cancer patients at two different times in disease progression and compared them with appropriately documented controls. As described below, this conceptually important application of a new profiling technique has led to both expected (cancer vs. control) results, but also an unprecedented finding of the gender (male vs. female) and cancer treatment (Cycle 1 vs Cycle 2) differences in the extent of glycosylation during cancer progression. The large standard deviations seen in Figure 1 represent inclusion of all data, presumably due to "biochemical individuality" of different patients. 
In assessing the profiling power of our procedure, we have included the sera of colorectal cancer patients from two different progressions of the disease (referred to as Cycle $1, n=38$, and Cycle 2, $n=24)$, and age-matched control individuals $(n=20)$ for comparative analyses. Both male and female patients have been included. From the selected 39 O-glycans (Table 1), all but two major components (m/z 879 and $\mathrm{m} / \mathrm{z} 1241)$ are less than $3 \%$ in abundance (Figure 1). These main $\mathrm{m} / \mathrm{z}$ values were structurally confirmed with MS/MS to represent sialyl-T (Neu-Gal-GalNAc) and disialyl T (Neu-Gal-(Neu)GalNAc) antigen, respectively (Figure S4, Supporting Information). Disialylated extended Core $2(\mathrm{~m} / \mathrm{z} 1690)$ was also confirmed with MS/MS (Figure S4, supporting information). This indicates that peaks observed in the O-glycan MALDI-MS spectrum (Figure S3) may represent extended Core 1 and Core $2 \mathrm{O}$-glycans in addition to extended Core 3 . Most minor glycans were detectable at below $0.5 \%$ abundance. The $\mathrm{T}$ antigen $(\mathrm{m} / \mathrm{z} 518$, Figure $\mathrm{S} 3$ ) was excluded from the calculations due to its unpredictable detection levels in both controls and cancer samples, a phenomenon that was not observed for any of the other 39 glycans that we describe in this communication. This was presumably due to interference from the lower molecular weight components of the MALDI matrix. We have previously observed that $\mathrm{N}$-glycans were not detected from fetuin using the same O-glycan release protocol (unpublished data). In the unlikely event that any remaining $\mathrm{N}$-glycans were to remain after PNGase $\mathrm{F}$ treatment and they were released together with the O-glycans during the permethylation treatment, they would be observed as high molecular weight peaks in the MALDI-MS spectrum. Of the 39 O-glycan peaks that we report in this study there is only one potential N-glycan overlap with the peak m/z 1836 which could potentially represent a small N-glycan (Table 1, peak 32). The glycan was not significantly altered in any of the statistical tests, however, and did not alter the conclusions of this study.

It appears that sialyl-T antigen and disialyl-T antigen are increasing in relative abundance as cancer progresses (i.e., comparing Cycle 2 to Cycle 1), while the reverse is true for the larger extended O-glycans (Figure 1). The decrease in the lower- abundance glycans is not due to the normalization of the data since the same trend was seen in the original spectra (a plot of non-normalized raw data is shown in Figure S5).

While there is a plausible agreement between the measured and computed $\mathrm{m} / \mathrm{z}$ values, the more definitive structures will need to be assigned by additional MS/MS fragmentation measurements, which, alas, could not be accomplished due to the very low signals for the minor profile components. We, however, feel encouraged that their detection became feasible as the result of the simultaneous cleavage/immediate permethylation in our protocol, which largely rules out a formation of artifacts. Sample enrichment procedures still need to be developed in future studies to secure MS/MS recordings for the minor glycans. However, our results are in fair agreement with the previous results from transcriptomic data ${ }^{31}$. While transcriptomic data may be considered less than definitive by today's technology standards, the general agreement with our suggested structures appears plausible. A selective advantage of these truncated structures seem to preclude other core structures and extended O-glycans to reach significant abundance numbers. The early sialylation precludes further extensions of the core structures. For readers' orientation with the structural relations of Core 1-4, see Supplementary Figure S6, Supporting Information. However, the relative depression of these 
structures is quite dramatic, correlating the disease in a statistically significant way as demonstrated in a battery of statistical tests indicated below.

For an initial statistical evaluation of the different cohort groups, we chose Principal Component Analysis (PCA). The PCA plots shown in Figure 2 indicate some basic trends: (A) different cohorts are not easily segregated when both males and females are included;

(B) for females alone, distinction is readily seen for both cancer cycles against controls; and (C) for males alone, a distinction is observed for Cycle 2 only when compared to the control and Cycle 1. Females and males thus appear to have distinct differences in how the glycan profiles correlate with disease progression.

Another means to determine if and how the data differ between the controls and cancer patients has been a one-way ANOVA test (at the $\mathrm{p}=0.05$ level), with Tukey means comparison test. While comparing the data obtained from 39 individual glycans as control vs. cancer (Cycle 1 and Cycle 2 combined), five glycan entries were deemed significant (Figure S7A, Supporting Information), but when judging the O-glycan level in different cycles, most entries were altered in Cycle 2 and only few in Cycle 1 (Figure S7B, Supporting Information). The ANOVA p-values and boxplots of cancer and control samples of the five significant glycans m/z $559\left(\mathrm{HexNAc}_{2}\right), 1212\left(\mathrm{Hex}_{3} \mathrm{HexNAc}_{2}\right), 1294\left(\mathrm{HexNAc}_{5}\right)$, $1703\left(\mathrm{Hex}_{2} \mathrm{HexNAc}_{5}\right)$ and $1865\left(\mathrm{Hex}_{4} \mathrm{HexNAc}_{4}\right)$ are shown in Figure 3.

A diagnostic value of individual glycans in the entire O-glycan profile was further assessed through the Receiver Operating Characteristic (ROC) evaluation, which is a widely recognized criterion to validate biomedical investigations ${ }^{35}$. In this test, the area-under-thecurve (AUC) values range from 0 to 1 , while $\mathrm{AUC}=0.5$ represents totally insignificant results; values between 0.7 to 0.8 , or 0.2 and 0.3 are viewed as "moderately accurate", values between 0.8 and 0.9 or 0.1 to 0.2 are viewed as "accurate" and, finally, values below 0.1 or above 0.9 are viewed as highly accurate. Values above and below 0.5 represent positive and negative correlation, respectively. The ROC curve AUC values for all 39 glycans (control vs. cancer (both cycles combined)) are shown in Figure S8 (Supporting Information). Four O-glycans display accurate AUC values below 0.2 (m/z 559, 1212, 1703, and 1865), 31 glycans are moderately accurate and four glycans (m/z 1050, 1241, 1690, and 1836) have ROC values that are not accurate (Figure S8, Supporting Information). Four of the five glycans plotted in Figure 3 are both significant in the one-way ANOVA test and deemed accurate by ROC under the curve test (m/z 559, 1212, 1703, and 1865), Figure 3.

All five significant O-glycans progressively decrease with the successive cancer cycles, with less relative abundance overlap with controls, lower p-values and better AUC values to support this notion. The PCA clusters (Figure 2) already indicated that there are genderspecific differences in the colorectal cancer disease progression. To investigate this further, we performed gender- and cycle-specific ROC analysis for all 39 glycans. In Cycle 1, the Oglycan serum profiles contain no accurate predictors of cancer in males, while the female profiles contain 10 highly accurate ROC AUC scores, 23 accurate and 2 moderately accurate scores (Figure S9, Supporting Information). In summary, the female O-glycan profiles change significantly already at Cycle 1 , while the male O-glycan profiles were not changed to the same extent. 
In Cycle 2, the ROC AUC predictive values of the O-glycans had increased dramatically in males (Figure 4). Twenty-one O-glycans were now highly accurate, 11 were accurate and 3 were moderately accurate. For females, the accuracy levels were now even higher in Cycle 2: 35 highly accurate ROC AUC values and four accurate ROC values (Figure 4). Our statistical tests indicate that the $\mathrm{O}$-glycosylation clearly correlates with disease progression. Some O-glycans even display AUC values of zero, indicating complete segregation between Cycle 2 and controls. For comparative purposes, the five O-glycans that were significant in Figure 3 (control vs cancer) are again plotted from the female Cycle 2 subgroup with boxplots and ROC curves now shown in Figure 5.

The value of our sample treatment and profiling protocol for the measurement and monitoring of serum O-glycans seems evident from the presented data and their statistical analyses: 39 glycans have been measurable and quantifiable in human serum, which is significantly more components than shown in any previous communication using MS-based techniques. Moreover, it is somewhat unclear as to how many glycans previously shown by others were actually procedural artifacts of beta-elimination.

Our MALDI-MS profiles indicate that increase of m/z 879 (sialyl-T antigen) and m/z 1241 (disialyl-T antigen) in blood serum is accompanied by a depression of numerous other Oglycans in the glycan profile. This is in contrast to what is observed in healthy human colon, where Core 1-4 are all expressed with Core 3 being the most abundant ${ }^{16}$. Up-regulation of both the sialyl $\mathrm{T}$ and disialyl $\mathrm{T}$ antigens is compliant with a strong expression of T-synthase (C1GalT1), ST3 beta-galactoside alpha-2,3-sialyltransferase 1 (ST3Gal-1) and ST6 Nacetylgalactosaminide alpha-2,6-sialyltransferase 1 (ST6GalNAc-1). Increase in disialyl-T antigen on serum hemopexin peptides was previously reported to be associated with colorectal cancer ${ }^{37}$. Low levels of Core 3 (GlcNAc $\beta 3$ GalNAc) and sialyl-Tn (Siaa6GalNAc) indicate that T-synthase competes effectively with Core $3 \beta 3$ GNT and ST6GalNAc I glycosyltransferases. The reciprocal relationship between these enzymes has also been shown in vitro ${ }^{38}$. Low or non-existent expression of Core 3 enzyme has previously been reported in colon cancers ${ }^{39}$.

Recently, it has also been shown that reduced Core 3 correlates with metastasis and poor prognosis of colorectal cancer patients by affecting MUC-1, P53 and miR-200c ${ }^{40}$. In addition, MUC-1 and MUC-2 expression seems to be dependent on Core 3 expression ${ }^{40,41}$. Down-regulation of MUC-1 and MUC-2 and other mucins has been reported to correlate with more aggressive cancers and poor prognosis ${ }^{42}$. Due to the small signals, we did not observe the Tn antigen (GalNAca, theoretical m/z 314) in the MALDI-MS spectra. This is to be expected due to strong matrix interference in the low $\mathrm{m} / \mathrm{z}$ range. Tn expression is strongly correlated with various cancers and not expressed in healthy tissues or, if so, at very low levels ${ }^{16}$. In our study, we observed sialyl Tn, STn ( $m / z$ 675, Siaa6GalNAc) that, interestingly, decreased with the disease progression. This is somewhat in contrast to previous reports from solid tumors, with the ratio of $\mathrm{Tn}$ to $\mathrm{T}$ antigen increased during cancer progression ${ }^{16}$. Strong expression of ST3Gal-1 blocks the production of Core 2 (GaLß3(GlcNAc $\beta 6$ )GalNAc), since Core 2 GNT1-2 competes with ST3Gal-1 over the Core 1 substrate and the two glycosyltransferases are known to co-localize ${ }^{43}$. In breast cancer, the dominant up-regulation of ST3Gal-1 is observed ${ }^{43}$. With low levels of Core 2, extended 
structures, such as polyLacNAc, are also notably down-regulated. This observation in serum is in contrast to what has been reported in colon cancer cell lines, where up-regulation of $\mathrm{C} 2 \mathrm{GnT} 1$ and down-regulation of $\mathrm{C} 2 \mathrm{GnT} 2$ were observed, resulting in up-regulation of Core 2 and Core 2-extended structures at the expense of Core 4 structures ${ }^{16}$.

The source of O-glycans that we detect in serum has not been determined as yet in the necessary detail. The $\mathrm{O}$-glycans may be derived from abundant proteins in serum, such as some O-glycosylated immunoglobulins or acute-phase proteins as well. Some O-glycans could also originate from inflammatory cells that are extensively coated with sialic acids. Moreover, it is also possible that our observed signals may originate from glycoproteins that have been released from the primary tumor. Only a limited number of glycoproteins have been reported to carry sialylated- or unsialylated T- and Tn-antigens in malignant tissues including MUC-1 and CD44V6 and osteopontin ${ }^{44}$. Several glycoprotein-based serum biomarkers have been used to differentiate cancer types. They are believed to be shedded from the tumor and are detected by monoclonal antibodies that recognize O-glycoproteins or O-glycans such as MUC-1 (CA15-3, breast cancer), MUC-16 (CA125, ovarian cancer), $\mathrm{SLe}^{\mathrm{a}}$ (CA19-9, pancreatic cancer), and STn (evaluated as biomarker for ovarian cancer) ${ }^{45}$. Transformed colon cancer tissue excrete glycoproteins, including mucins, to the circulation in contrast to healthy differentiated tissue ${ }^{45}$. Thus, the glycans that we detect could originate from the solid tumor. In all regards, the total glycan profile of the serum should not be expected to be entirely the same as the profile of the solid tumor, even though some overlap is theoretically possible.

The altered "glycan coat" on tumor cells may be immunomodulatory, which is something that has raised interest recently ${ }^{46}$. Sialic acid-binding immunoglobulin-like lectins (SIGLECs), macrophage galactose-specific lectin (MGL) and dendritic cell (DC)-specific ICAM-3-grabbing non-integrin 1 (DC-SIGN/CD209) are expressed on immune cells and can mediate immune suppression by distinct pathways through binding to specific glycans covering tumor cells ${ }^{46}$. It has been suggested that the altered glycosylation in cancer may be a novel immune checkpoint, suitable as a therapeutic target ${ }^{46}$.

While differences in the onset of O-glycosylation between males and females as observed in our study are striking, our exhaustive literature search did not reveal any studies in connection with hormonal regulation of O-glycosylation effects that would justify the reason behind this observation. Besides the well-known studies on pregnancy-associated changes in glycodelin ${ }^{47}$ and uromodulin ${ }^{48}$ conducted in the laboratories of Howard Morris and Ann Dell two decades ago, the literature on hormonally-dependent N-glycosylation is not particularly abundant either. The relatively recent reports include post-translationallyinduced functional targeting of the hormone thyrotropin ${ }^{49}, \mathrm{~N}$-glycome changes in plasma during pregnancy ${ }^{50}$, and the sex-related differences in $\mathrm{N}$-glycosylation of $\mathrm{IgG}$ in both humans and mice ${ }^{51-53}$. We believe the knowledge discrepancies between $\mathrm{N}$-glycosylation and $\mathrm{O}$-glycosylation is largely attributable to different methodological difficulties in the sample treatment for $\mathrm{N}$-versus O-glycans, respectively. Our observations show that the serum O-glycan profiles correlate strongly with cancer progression and display marked gender differences. Even if the observation of aberrant O-glycosylation in the serum profile of a late stage cancer is of limited prognostic value, these observations should encourage the 
research community to investigate more extensively the biological roles of O-glycosylation in cancer.

\section{CONCLUSIONS}

A new analytical protocol for the recovery of O-glycans from minute volumes of blood serum has been established and validated through MALDI-MS profiling of 39 glycans in 20 control and 62 colorectal cancer serum samples. The nearly simultaneous alkaline-based Oglycan cleavage from serum glycoproteins and a quantitative permethylation is the key to preserving the integrity of minor glycans and their quantitative profiling. The following statistical evaluation of the acquired O-glycan profiles from different colorectal cancer patient cohorts shows substantial agreement with the previously reported or suspected glycan structures extracted from either tissue biopsies or cancer cell lines. While our data obtained from the already advanced cancer cases do not yield any potential diagnostic biomarkers, they still indicate some promise for prognostic measurements using relatively non-invasive blood samples. A most remarkable outcome of our profiling measurements has been the finding of gender-dependent $\mathrm{O}$-glycosylation during cancer progression. Whereas no explanations of this can be provided at this time, this finding, together with the availability of the described protocol and methodology, should stimulate further research in this area.

\section{Supplementary Material}

Refer to Web version on PubMed Central for supplementary material.

\section{ACKNOWLEDGMENTS}

This work was supported by the grants from the National Institute of General Medical Sciences, U.S. Department of Health (NIH R21GM118340 and NIH R01GM106084). We thank Hoosier Oncology Group for providing the serum samples and clinical information on all cancer patients. These activities were, in turn, supported under Grant U01 CA128535 from the National Cancer Institute. Reporting on experimental details has been prepared in accordance with MIRAGE (The Minimum Information Required for a Glycomics Experiment) sample preparation (dx.doi.org/10.3762/mirage.1) and MS guidelines (dx.doi.org/10.3762/mirage.2).

\section{REFERENCES}

(1). Varki A, Kannagi R, Toole B, et al. In Essentials of Glycobiology; Varki A, Cummings RD, Esko JD, et al., Ed.; Cold Spring Harbor Laboratory Press: Cold Spring Harbor (NY), 2017; p Chapter 47.

(2). Hakomori S-I Cancer Res. 1985, 45 (6), 2405-2414. [PubMed: 3886132]

(3). Hakomori S Proc. Natl. Acad. Sci. U. S. A 2002, 99 (16), 10231-10233. [PubMed: 12149519]

(4). Ribeiro JP; Mahal LK Curr. Opin. Chem. Biol 2013, 17 (5), 827-831. [PubMed: 23856055]

(5). Novotny MV; Alley WR Curr. Opin. Chem. Biol 2013, 17 (5), 832-840. [PubMed: 23790311]

(6). Alley WR; Mann BF; Novotny MV Chem. Rev 2013, 113 (4), 2668-2732. [PubMed: 23531120]

(7). Xia L; Gildersleeve JC Methods Mol. Biol 2015, 1331, 27-40. [PubMed: 26169733]

(8). Surinova S; Schiess R; Hüttenhain R; Cerciello F; Wollscheid B; Aebersold R J. Proteome Res 2011, 10 (1), 5-16. [PubMed: 21142170]

(9). Alley WR Jr; Vasseur JA; Goetz JA; Svoboda M; Mann BF; Matei DE; Menning N; Hussein A; Mechref Y; Novotny MV J Proteome Res 2012, 11 (4), 2282-2300. [PubMed: 22304416] 
(10). Vasseur JA; Goetz JA; Alley WR; Novotny MV Glycobiology 2012, 22 (12), 1684-1708. [PubMed: 22781126]

(11). Mann BF; Goetz JA; House MG; Schmidt CM; Novotny MV Mol. Cell. Proteomics 2012, 11 (7), M111.015792.

(12). Holst S; Deuss AJM; van Pelt GW; van Vliet SJ; Garcia-Vallejo JJ; Koeleman CAM; Deelder AM; Mesker WE; Tollenaar RA; Rombouts Y; Wuhrer M Mol. Cell. Proteomics 2016, 15 (1), 124-140. [PubMed: 26537799]

(13). Biskup K; Braicu EI; Sehouli J; Fotopoulou C; Tauber R; Berger M; Blanchard VJ Proteome Res. 2013, 12 (9), 4056-4063.

(14). Carlson DM J. Biol. Chem. 1968, 243 (3), 616-626. [PubMed: 5637714]

(15). Maho Amano MT J. Glycomics Lipidomics 2013, 03 (01), 1-8.

(16). Brockhausen I EMBO Rep. 2006, 7 (6), 599-604. [PubMed: 16741504]

(17). Tarp MA; Clausen H Biochim. Biophys. Acta 2008, 1780 (3), 546-563. [PubMed: 17988798]

(18). Hollingsworth MA; Swanson BJ Nat. Rev. Cancer 2004, 4 (1), 45-60. [PubMed: 14681689]

(19). Goetz JA; Novotny MV; Mechref Y Anal. Chem 2009, 81 (23), 9546-9552. [PubMed: 19874002]

(20). Snyder CM; Alley WR; Campos MI; Svoboda M; Goetz JA; Vasseur JA; Jacobson SC; Novotny MV Anal. Chem 2016, 88 (19), 9597-9605. [PubMed: 27575585]

(21). Ferlay J; Steliarova-Foucher E; Lortet-Tieulent J; Rosso S; Coebergh JWW; Comber H; Forman D; Bray F Eur. J. Cancer 2013, 49 (6), 1374-1403. [PubMed: 23485231]

(22). Schmoll HJ; Van Cutsem E; Stein A; Valentini V; Glimelius B; Haustermans K; Nordlinger B; van de Velde CJ; Balmana J; Regula J; Nagtegaal ID; Beets-Tan RG; Arnold D; Ciardiello F; Hoff P; Kerr D; Köhne CH; Labianca R; Price T; Scheithauer W; Sobrero A; Tabernero J; Aderka D; Barroso S; Bodoky G; Douillard JY; El Ghazaly H; Gallardo J; Garin A; Glynne-Jones R; Jordan K; Meshcheryakov A; Papamichail D; Pfeiffer P; Souglakos I; Turhal S; Cervantes A Ann. Oncol. Off. J. Eur. Soc. Med. Oncol 2012, 23 (10), 2479-2516.

(23). Holst S; Wuhrer M; Rombouts Y Adv. Cancer Res 2015, 126, 203-256. [PubMed: 25727149]

(24). Pinho SS; Reis CA Nat. Rev. Cancer 2015, 15 (9), 540-555. [PubMed: 26289314]

(25). Dalziel M; Crispin M; Scanlan CN; Zitzmann N; Dwek RA Science 2014, 343 (6166), 1235681. [PubMed: 24385630]

(26). Dube DH; Bertozzi CR Nat. Rev. Drug Discov. 2005, 4 (6), 477-488. [PubMed: 15931257]

(27). Christiansen MN; Chik J; Lee L; Anugraham M; Abrahams JL; Packer NH Proteomics 2014, 14 (4-5), 525-546. [PubMed: 24339177]

(28). Williams TI; Saggese DA; Muddiman DC J. Proteome Res 2008, 7 (6), 2562-2568. [PubMed: 18422354]

(29). Kirmiz C; Li B; An HJ; Clowers BH; Chew HK; Lam KS; Ferrige A; Alecio R; Borowsky AD; Sulaimon S; Lebrilla CB; Miyamoto S Mol. Cell. Proteomics 2007, 6 (1) 43-55. [PubMed: 16847285]

(30). An HJ; Miyamoto S; Lancaster KS; Kirmiz C; Li B; Lam KS; Leiserowitz GS; Lebrilla CB J. Proteome Res 2006, 5 (7), 1626-1635. [PubMed: 16823970]

(31). Vavasseur F; Dole K; Yang J; Matta KL; Myerscough N; Corfield A; Paraskeva C; Brockhausen I Eur. J. Biochem 1994, 222 (2), 415-424. [PubMed: 8020479]

(32). Miura Y; Kato K; Takegawa Y; Kurogochi M; Furukawa J-I; Shinohara Y; Nagahori N; Amano M; Hinou H; Nishimura S-I Anal. Chem 2010, 82 (24), 10021-10029. [PubMed: 21077635]

(33). Yoshida Y; Furukawa J-I; Naito S; Higashino K; Numata Y; Shinohara Y Proteomics 2016, 16 (21), 2747-2758. [PubMed: 27601404]

(34). Furukawa J; Piao J; Yoshida Y; Okada K; Yokota I; Higashino K; Sakairi N; Shinohara Y Anal Chem 2015, 87 (15), 7524-7528. [PubMed: 26132640]

(35). Zweig MH; Campbell G Clin. Chem. 1993, 39 (4), 561-577. [PubMed: 8472349]

(36). Varki A; Cummings RD; Esko JD; Freeze HH; Stanley P; Marth JD; Bertozzi CR; Hart GW; Etzler ME Proteomics 2009, 9 (24), 5398-5399. [PubMed: 19902428] 
(37). Darebna P; Novak P; Kucera R; Topolcan O; Sanda M; Goldman R; Pompach PJ. Proteomics 2016, 153, 44-52. [PubMed: 27646713]

(38). Barrow H; Tam B; Duckworth CA; Rhodes JM; Yu LG PLoS One 2013, 8 (3), 1-6.

(39). Brockhausen I Biochim. Biophys. Acta - Gen. Subj. 1999, 1473 (1), 67-95.

(40). Ye J; Wei X; Shang Y; Pan Q; Yang M; Tian Y; He Y; Peng Z; Chen L; Chen W; Wang R Oncogene 2017, 36 (46), 6391-6407. [PubMed: 28745318]

(41). Xia L Methods Enzymol. 2010, 479 (10), 123-141. [PubMed: 20816163]

(42). Behera SK; Praharaj AB; Dehury B; Negi S Glycoconj. J 2015, 32 (8), 575-613. [PubMed: 26239922]

(43). Dalziel M; Whitehouse C; McFarlane I; Brockhausen I; Gschmeissner S; Schwientek T; Clausen H; Burchell JM; Taylor-Papadimitriou J J. Biol. Chem 2001, 276 (14), 11007-11015. [PubMed: 11118434]

(44). Häuselmann I; Borsig L Front. Oncol 2014, 4 (2), 1-15. [PubMed: 24478982]

(45). Kudelka MR; Ju T; Heimburg-Molinaro J; Cummings RD Adv. Cancer Res. 2015, 126, 53-135. [PubMed: 25727146]

(46). RodrÍguez E; Schetters STT; van Kooyk Y Nat. Rev. Immunol 2018, 18 (3), 204-211. [PubMed: 29398707]

(47). Morris HR; Dell A; Easton RL; Panico M; Koistinen H; Koistinen R; Oehninger S; Patankar MS; Seppala M; Clark GFJ. Biol. Chem 1996, 271 (50), 32159-32167. [PubMed: 8943270]

(48). Easton RL; Patankar MS; Clark GF; Morris HR; Dell AJ. Biol. Chem 2000, 275 (29), 21928 21938. [PubMed: 10770931]

(49). Ikegami K; Liao XH; Hoshino Y; Ono H; Ota W; Ito Y; Nishiwaki-Ohkawa T; Sato C; Kitajima K; Iigo M; Shigeyoshi Y; Yamada M; Murata Y; Refetoff S; Yoshimura T Cell Rep. 2014, 9 (3), 801-809. [PubMed: 25437536]

(50). Ruhaak LR; Uh HW; Deelder AM; Dolhain RE; Wuhrer M J. Proteome Res 2014, 13 (3),16571668. [PubMed: 24527664]

(51). Chen G; Wang Y; Qiu L; Qin X; Liu H; Wang X; Wang Y; Song G; Li F; Guo Y; Li F; Guo S; Li ZJ. Proteomics 2012, 75 (10), 2824-2834. [PubMed: 22365975]

(52). Melanson E; Bombardier C; Saraux A; Combe B; Dougados M; Bengtsson C; Hausmann JS; Campbell H; Kaiser UB; Rudd PM; Lauc G; Wilson JF; Finkelstein JS; Nigrovic PAJ Appl Physiol 2017, 119 (9), 975-981.

(53). Krištić J; Zaytseva OO; Ram R; Nguyen Q; Novokmet M; Vučković F; Vilaj M; TrbojevićAkmačić I; Pezer M; Davern KM; Morahan G; Lauc G Nat. Chem. Biol. 2018, 14 (5), 516-524. [PubMed: 29632412] 

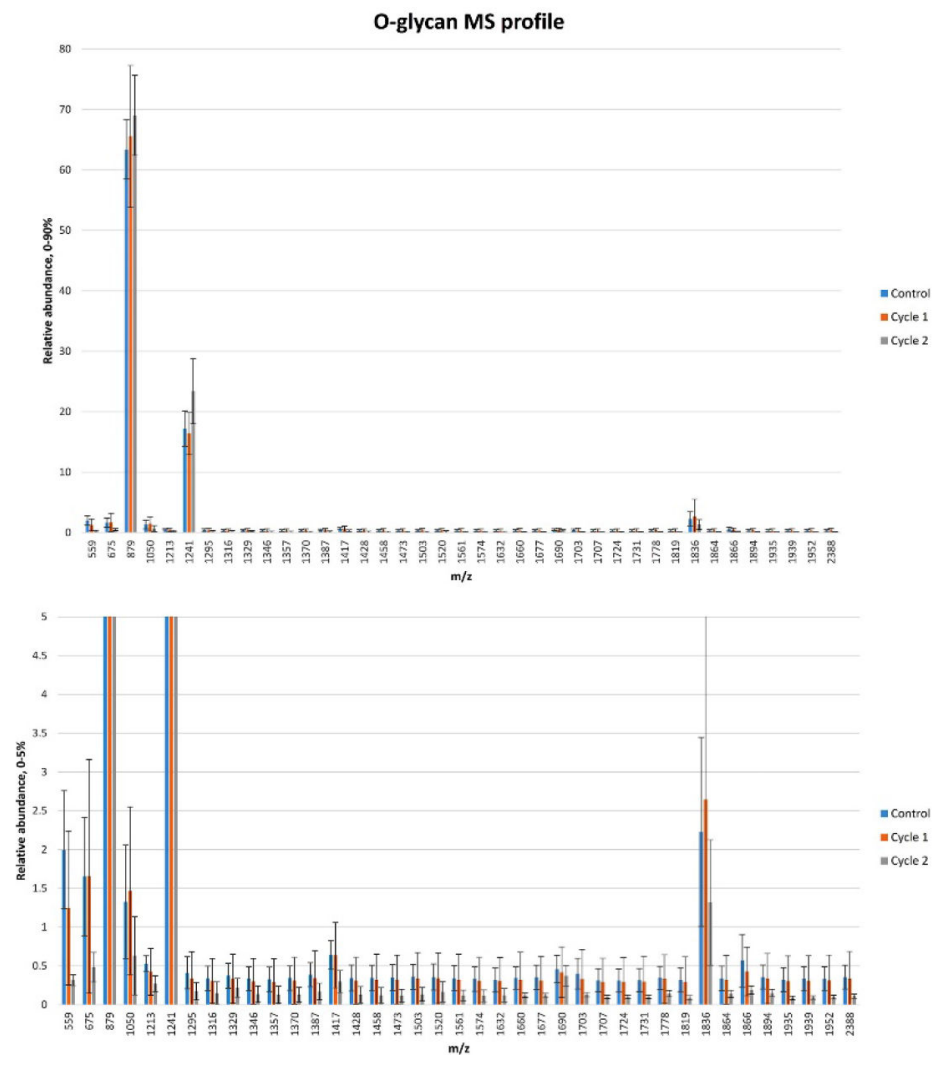

Figure 1.

A comprehensive serum O-glycan profile derived from control and colorectal cancer Cycle 1 and Cycle 2 samples. The relative abundance of 39 permethylatedO-glycanswas determined using MALDI-MS and the in-house developed software PeakCalc. With the expected repeatability of the MALDI-MS-based measurements (approximately $\pm 15 \%$ variation), the large standard deviation error bars very likely reflect substantial variations due to a disease state and possibly gender. 

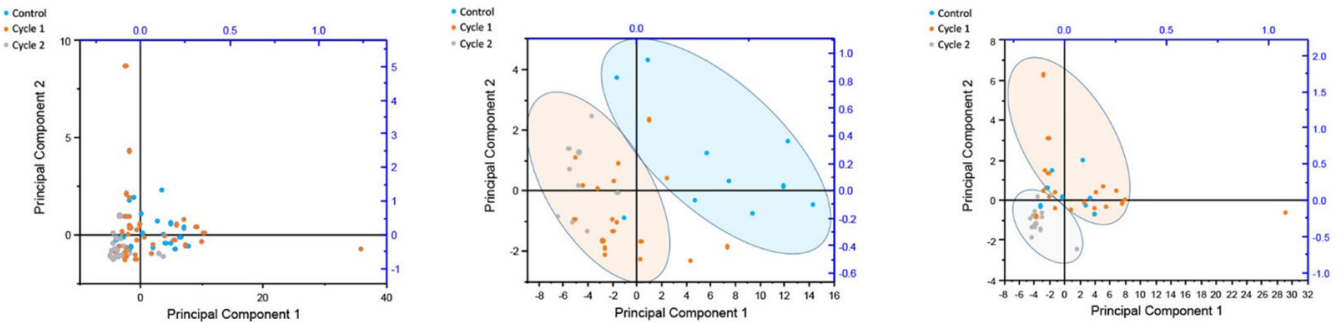

Figure 2.

PCA plot of principal component 1 and 2 of 39 O-glycansfrom three different cancer conditions: control (blue), Cycle 1 (orange), and Cycle 2 (gray). The groups are not easily segregated when both males and females are included (A). For females the PCA segregates both cancer cycles from the control (B), while the PCA of males segregate Cycle 2 from the control and Cycle 1(C). 


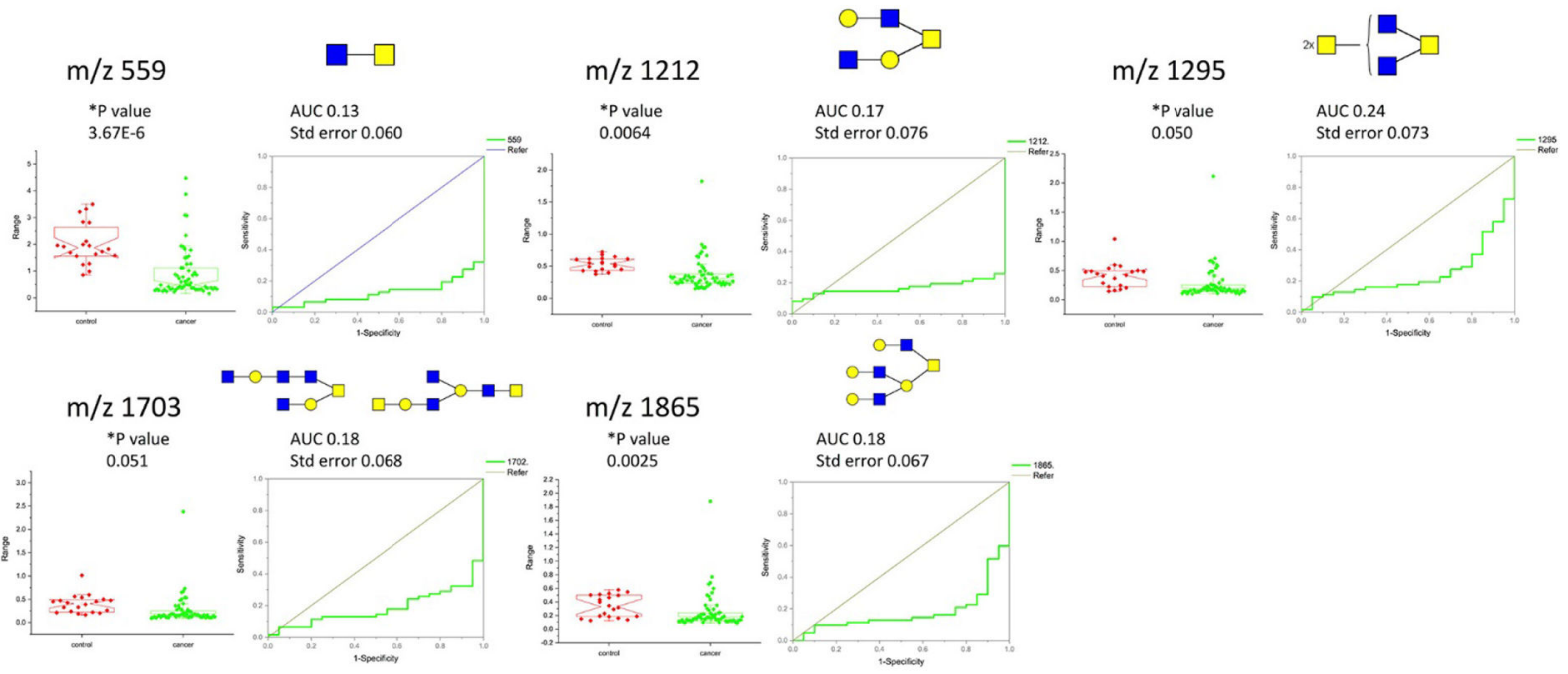

Figure 3.

Boxplots of O-glycanssignificantly altered in cancer as determined with one-way ANOVA (at appropriate $\mathrm{p}=0.05$ values) and ROC curves with AUC values and associated standard errors. The low AUC values represent strong negative correlation. Carbohydrate representations are drawn according to symbol nomenclature as suggested by Varki et al. ${ }^{36}$ 


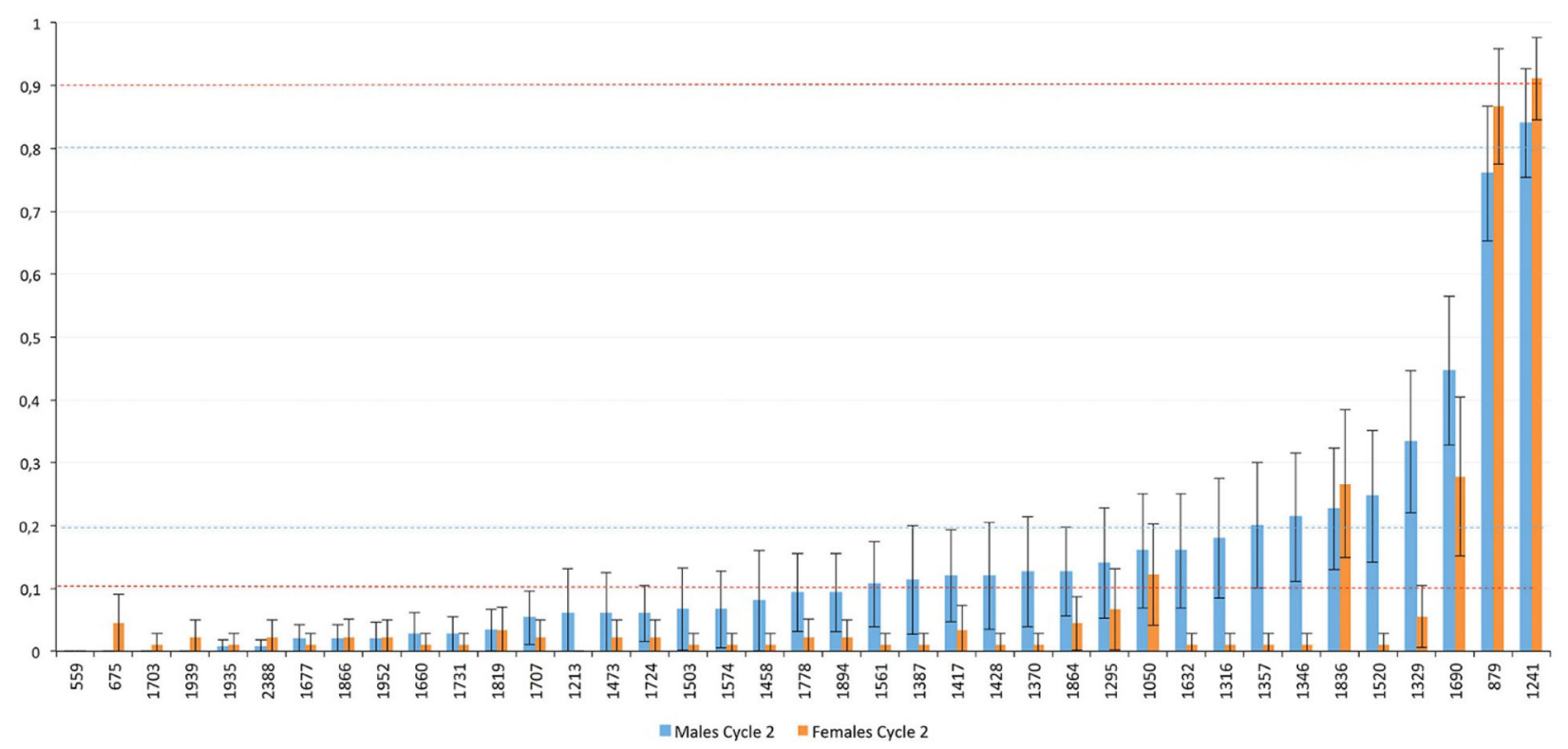

Figure 4.

ROC AUC values of controls versus Cycle 2 of 39 serum O-glycans. Female (orange) and male (blue) data bars are plotted separately. The error bars denote standard error of the AUC plots. The glycans are listed in increasing ROC area order. Bars below 0.2 orabove 0.8 are considered accurate, while values below 0.1 or above 0.9 are highly accurate. The lower values indicate decreased expression in cancer, while the positive values represent higher expression in cancer. 

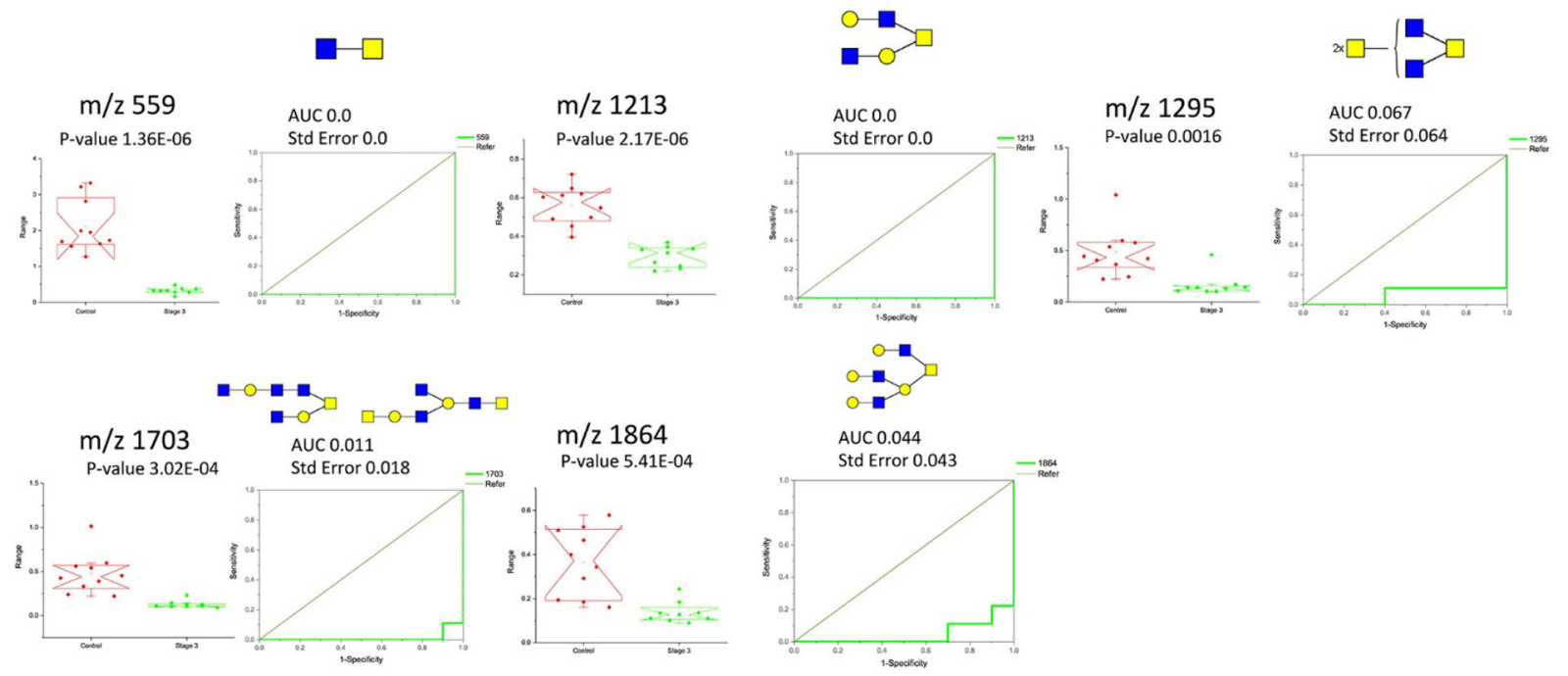

Figure 5.

Female Cycle $2 \mathrm{O}$-glycansdisplay strong statistical change compared to controls as demonstrated by one-way ANOVA and ROC AUC plots with associated standard error values. 
Table 1.

Compositions of 39 serum O-glycans. The O-glycans were released and permethylated prior to MALDI-MS detection. Theoretical monoisotopic masses are listed side-by-side with the observed values.

\begin{tabular}{|c|c|c|c|}
\hline Peak ID & Presumptive composition & $\begin{array}{l}\text { Observed mass } \\
{[\mathrm{M}+\mathrm{Na}]+}\end{array}$ & $\begin{array}{l}\text { GlycoMod expected } \\
\text { mass }[\mathrm{M}+\mathrm{Na}]+\end{array}$ \\
\hline 1 & $(\mathrm{HexNAc}) 2$ & 559.3 & 559.25 \\
\hline 2 & $(\mathrm{HexNAc}) 1(\mathrm{NeuAc}) 1$ & 675.4 & 675.27 \\
\hline 3 & (Hex)1(HexNAc)1(NeuAc) 1 & 879.4 & 879.38 \\
\hline 4 & (HexNAc) 4 & 1049.6 & 1049.62 \\
\hline 5 & (Hex)2(HexNAc)3 & 1212.6 & 1212.64 \\
\hline 6 & (Hex)1(HexNAc)2(NeuAc)2 & 1240.6 & 1240.56 \\
\hline 7 & $(\mathrm{HexNAc}) 5$ & 1294.6 & 1294.79 \\
\hline 8 & (Hex)2(HexNAc)2(dHex)2 & 1315.6 & 1315.80 \\
\hline 9 & $(\mathrm{Hex}) 2(\mathrm{HexNAc}) 2(\mathrm{NeuAc}) 1$ & 1328.6 & 1328.81 \\
\hline 10 & (Hex)3(HexNAc)2(dHex)1 & 1345.7 & 1345.87 \\
\hline 11 & (Hex)1(HexNAc)3(dHex)2 & 1356.7 & 1356.83 \\
\hline 12 & (Hex)1(HexNAc)3(NeuAc)1 & 1369.7 & 1369.76 \\
\hline 13 & (Hex)2(HexNAc)3(dHex)1 & 1386.7 & 1386.80 \\
\hline 14 & $(\mathrm{Hex}) 3(\mathrm{HexNAc}) 3$ & 1416.7 & 1416.68 \\
\hline 15 & (Hex)1(HexNAc)4(dHex)1 & 1427.7 & 1427.82 \\
\hline 16 & (Hex)2(HexNAc)4 & 1457.7 & 1457.86 \\
\hline 17 & (Hex)1(HexNAc)2(dHex)1(NeuAc) 1 & 1472.7 & 1472.92 \\
\hline 18 & (Hex)2(HexNAc)2(dHex)1(NeuAc)1 & 1502.7 & 1502.86 \\
\hline 19 & (Hex)3(HexNAc)2(dHex)2 & 1519.8 & 1519.90 \\
\hline 20 & (Hex)2(HexNAc)3(dHex)2 & 1560.8 & 1560.89 \\
\hline 21 & $(\mathrm{Hex}) 2(\mathrm{HexNAc}) 3(\mathrm{NeuAc}) 1$ & 1573.8 & 1573.89 \\
\hline 22 & (Hex)2(HexNAc)4(dHex)1 & 1631.8 & 1631.94 \\
\hline 23 & (Hex)1(HexNAc)2(dHex)1(NeuAc)2 & 1659.9 & 1659.78 \\
\hline 24 & (Hex)2(HexNAc)2(dHex)2(NeuAc)1 & 1676.8 & 1678.87 \\
\hline 25 & $(\mathrm{Hex}) 2(\mathrm{HexNAc}) 2(\mathrm{NeuAc}) 2$ & 1689.8 & 1689.81 \\
\hline 26 & $(\mathrm{Hex}) 2(\mathrm{HexNAc}) 5$ & 1702.8 & 1702.91 \\
\hline 27 & (Hex)3(HexNAc)2(dHex)1(NeuAc)1 & 1706.8 & 1706.94 \\
\hline 28 & $(\mathrm{Hex}) 4(\mathrm{HexNAc}) 2(\mathrm{dHex}) 2$ & 1723.9 & 1723.94 \\
\hline 29 & (Hex)1(HexNAc)3(NeuAc)2 & 1730.9 & 1730.93 \\
\hline 30 & $(\mathrm{Hex}) 3(\mathrm{HexNAc}) 3(\mathrm{NeuAc}) 1$ & 1777.8 & 1777.84 \\
\hline 31 & (Hex)2(HexNAc)4(NeuAc)1 & 1818.9 & 1819.03 \\
\hline 32 & (Hex)3(HexNAc)4(dHex)1 & 1835.9 & 1835.86 \\
\hline 33 & (Hex)2(HexNAc)2(dHex)1(NeuAc)2 & 1863.9 & 1863.93 \\
\hline 34 & (Hex)4(HexNAc) 4 & 1865.9 & 1865.90 \\
\hline 35 & (Hex)3(HexNAc)2(NeuAc)2 & 1893.9 & 1893.92 \\
\hline
\end{tabular}




\begin{tabular}{|l|l|l|l|}
\hline Peak ID & Presumptive composition & $\begin{array}{l}\text { Observed mass } \\
{[\mathbf{M}+\mathrm{Na}]+}\end{array}$ & $\begin{array}{l}\text { GlycoMod expected } \\
\text { mass [M +Na]+ }\end{array}$ \\
\hline 36 & (Hex)2(HexNAc)3(NeuAc)2 & 1935.0 & 1934.98 \\
\hline 37 & (Hex)3(HexNAc)3(dHex)3 & 1938.9 & 1939.03 \\
\hline 38 & (Hex)3(HexNAc)3(dHex)1(NeuAc)1 & 1952.0 & 1951.89 \\
\hline 39 & (Hex)4(HexNAc)4(dHex)3 & 2388.1 & 2388.06 \\
\hline
\end{tabular}

